\title{
ON GROUPS WHICH ARE RESIDUALLY $\mathfrak{X}$-COMMUTATIVE
}

\author{
by DAVID G. ARRELL
}

(Received 14th June 1974)

\section{Introduction and notation}

In this paper we study a property which we call residual $\mathfrak{X}$-commutativity. This idea was kindled by a paper of Ayoub (1) and one of Stanley (6). Durbin has defined a similar property in (2) which has also been studied by Slotterbeck in (4). Durbin's property implies residual $\mathfrak{X}$-commutativity but we have not been able to decide if they are equivalent. However, we have shown that they coincide in certain circumstances. The notation, unless otherwise stated, is that of Robinson (3).

Let $\mathfrak{X}$ be any class of groups. We define the family $\mathscr{H}(G: \mathfrak{X})$ of normal subgroups of $G$ by

$$
\mathscr{H}(G: \mathfrak{X})=\{N \triangleleft G \mid G / N \in \mathfrak{X}\}
$$

and the $\mathfrak{X}$-residual of a group $G$ by

$$
\rho_{\mathfrak{x}}^{*}(G)=\cap\{N \mid N \in \mathscr{H}(G: \mathfrak{X})\} .
$$

Following Stanley (5), we define the $\mathfrak{X}$-centre $H_{1}(G: \mathfrak{X})$ of a group $G$ by

$$
H_{1}(G: \mathfrak{X})=\{x \in G \mid(\exists N \in \mathscr{H}(G: \mathfrak{X}))[x, N]=1\}
$$

so if $\mathfrak{X}$ is a variety, for example, $H_{1}(G: \mathfrak{X})$ is the centraliser of the $\mathfrak{X}$-verbal subgroup in $G$. We say that $G$ is $\mathfrak{X}$-abelian if $G=H_{1}(G: \mathfrak{X})$.

We say that $\Omega=\left\{W_{\sigma}, V_{\sigma} \mid \sigma \in \Sigma\right\}$ is a normal $\mathfrak{X}$-factor covering of a group $G$ if

(i) for all $\sigma \in \Sigma, W_{\sigma} \triangleleft G, V_{\sigma} \triangleleft G$ and $V_{\sigma} \leqq W_{\sigma}$,

(ii) whenever $1 \neq g \in G$, there exists $\sigma \in \Sigma$ such that $g \in W_{\sigma}-V_{\sigma}$ and

(iii) for all $\sigma \in \Sigma, W_{\sigma} / V_{\sigma} \in \mathfrak{X}$.

We denote by $\mathfrak{X}^{\dagger}$ the class of groups which have a normal $\mathfrak{X}$-factor covering. If, in addition to (i), (ii) and (iii) above, we have

(iv) whenever $\lambda<\sigma, W_{\lambda} \leqq V_{\sigma}$

we say that $\Omega$ is a normal $\mathfrak{X}$-series for $G$ and denote by $\hat{\boldsymbol{P}}_{n} \mathfrak{X}$ the class of groups which have a normal $\mathfrak{X}$-series.

We shall use the following closure properties of a class $\mathfrak{X}$ :

a class $\mathfrak{X}$ is $\boldsymbol{H}$-closed if and only if every homomorphic image of an $\mathfrak{X}$-group is an $\mathfrak{X}$-group and we write $\mathfrak{X}=\boldsymbol{H} \mathfrak{X}$;

a class $\mathfrak{X}$ is $S_{n}$-closed if and only if every normal subgroup of an $\mathfrak{X}$-group is an $\mathfrak{X}$-group and we write $\mathfrak{X}=S_{n} \mathfrak{X}$. 
The property introduced by Ayoub in (1), which we shall call residual commutativity, is defined by

a group $G$ is residually commutative if and only if whenever $a$ and $b$ are non-trivial elements of $G$, there exists $M$ normal in $G$ such that $M$ contains $[a, b]$ but not both $a$ and $b$.

Following Stanley in (6) we say that a group $G$ is residually $\mathfrak{X}$-central if and only if whenever $1 \neq g \in G$, there exists $H$ normal in $G$ such that $H$ does not contain $g$ but $g H \in H_{1}(G / H: \mathfrak{X})$. We denote by $\mathfrak{X}$ the class of residually $\mathfrak{X}$-central groups.

We now define the relation $\mathscr{C}(g, h, M)$ on elements $g$ and $h$ of a group $G$ and a normal subgroup $M$ of $G$ by

$\mathscr{C}(g, h, M)$ if and only if $M$ contains $[g, h]$ but not both $g$ and $h$ and the relation $\mathscr{R}(g, H, N)$ on the element $g$ of $G$, a normal subgroup $H$ of $G$ and $N \in \mathscr{H}(H: \mathfrak{X})$ by

$\mathscr{R}(g, H, N)$ if and only if $g \in H$ and whenever $1 \neq h \in N$ there exists $M$ normal in $G$ such that $\mathscr{C}(g, h, M)$.

We are now able to define the classes $\mathfrak{X}^{(0)}$ and $\mathfrak{X}^{(1)}$ by

$G \in \mathfrak{X}^{(0)}$ if and only if for all $1 \neq g \in G$, there exists $N \in \mathscr{H}(G: \mathfrak{X})$ such that $\mathscr{R}(g, G, N)$;

$G \in \mathfrak{X}^{(1)}$ if and only if for all $1 \neq g \in G$, there exist $H$ normal in $G$ and $N \in \mathscr{H}(H: \mathfrak{X})$ such that $\mathscr{R}(g, H, N)$;

and we say that $G$ is residually $\mathfrak{X}$-commutative if and only if $G \in \mathfrak{X}^{(1)}$. Finally, we define $\mathfrak{M}_{n}^{\vee}$ to be the class of groups which possess the minimal condition on normal subgroups.

\section{Statement of theorems}

Theorem A. Let $G$ be a group which possesses the minimal condition on normal subgroups and let $\mathfrak{X}=S_{n} \mathfrak{X}=H \mathfrak{X}$. The following conditions are equivalent:

(i) $G$ is residually $\mathfrak{X}$-commutative,

(ii) $G$ has a normal $\mathfrak{X}$-abelian factor covering,

(iii) $G$ has an ascending normal series with $\mathfrak{X}$-abelian factors.

The proof of (ii) if and only if (iii) is due to Durbin (2).

Theorem B. If $\mathfrak{X}$ is a variety of groups then the class of residually $\mathfrak{X}$-commutative groups is a local class.

\section{Basic lemmas}

Let $\mathfrak{X}$ be an arbitrary class of groups. 
Lemma 3.1. $\mathfrak{X}^{(1)}=\mathfrak{X}^{(0)}$ when $\mathfrak{X}$ is the trivial class, with inequality in general, even when $\mathfrak{X}$ is a variety.

Proof. Let $\mathbb{E}$ denote the trivial class of groups. It is clear from the definitions that $\mathfrak{F}^{(0)} \leqq \mathfrak{E}^{(1)}$ and so we need only show the inclusion $\mathfrak{E}^{(1)} \leqq \mathfrak{E}^{(0)}$. Let $G \in \mathbb{F}^{(1)}$ and $a$ and $b$ be two non-trivial elements of $G$. Since $G$ is residually E-commutative, we can find $H$ normal in $G$ such that $\mathscr{R}(a, H, H)$; if $b$ does not belong to $H$ then $\mathscr{C}(a, b, H)$ and $\mathscr{R}(a, G, G)$. If $b \in H$, then there exists $M$ normal in $G$ such that $\mathscr{C}(a, b, M)$ and again $\mathscr{R}(a, G, G)$. In both cases we have shown that $G \in \mathbb{E}^{(0)}$.

To show that $\mathfrak{X}^{(0)}<\mathfrak{X}^{(1)}$, consider the following example. Let $\mathfrak{X}$ be the variety generated by $A_{5}$, the alternating group on five letters. Define

$$
H^{(0)}=1
$$

and

$$
H^{(n+1)}=H^{(n)} \chi A_{5}, \quad(n \geqq 0)
$$

the wreath product.

We can identify $H^{(n-1)}$ with one of the direct factors of the base group of $H^{(n)}$ and so obtain a series

$$
1=H^{(0)} \leqq H^{(1)} \leqq H^{(2)} \leqq \ldots \leqq H^{(n)} \leqq \ldots
$$

and let $G=\bigcup_{n=1}^{\infty} H^{(n)}$. Let $N^{(n)}$ be the normal closure of $H^{(n)}$ in $G$; then $G=\bigcup_{n=1}^{\infty} N^{(n)}$ and $N^{(n+1)} / N^{(n)} \in \mathfrak{X}$, which shows that $G$ has a normal ascending series with $\mathfrak{X}$-factors and hence is residually $\mathfrak{X}$-commutative.

We now examine the $\mathfrak{X}$-verbal subgroups $W\left(H^{(n)}\right)$. Since

$$
W\left(H^{(1)}\right)=1=\left(H^{(0)}\right)^{H^{(1)}}
$$

we take as inductive hypothesis

$$
W\left(H^{(k)}\right)=\left(H^{(k-1)}\right)^{H^{(k)}}, \quad \text { for } k=1,2,3, \ldots, n-1
$$

and let $N$ denote the normal closure of $H^{(1)}$ in $H^{(n)}$. Then, as

jt follows that

$$
H^{(n)} / N \cong H^{(n-1)}
$$

and in particular

$$
W\left(H^{(n)} / N\right) \cong\left(H^{(n-2)}\right)^{H^{(n-1)}}
$$

$$
\frac{W\left(H^{(n)}\right) N}{N}=\frac{\left(H^{(n-1)}\right)^{H^{(n)}} N}{N} .
$$

But $N$ is contained in $\left(H^{(n-1)}\right)^{H^{(n)}}$ and $W\left(H^{(n)}\right)$ and thus

$$
W\left(H^{(n)}\right)=\left(H^{(n-1)}\right)^{H^{(n)}}
$$

as required. This implies that $H^{(n-1)} \leqq W\left(H^{(n)}\right)$. 
We now assert that $W(G)=G$; for if $g \in G$, then $g \in H^{(n)}$, for some integer $n$, and so $g \in W\left(H^{(n+1)}\right) \leqq W(G)$. Thus the only choice for $N$ in the $\mathfrak{X}^{(0)}$ definition is $N=G$. Let $a$ and $b$ be two non-commuting elements of $H^{(1)}=A_{5}$. It is then clear that $G$ cannot satisfy the $\mathfrak{X}^{(0)}$ definition.

Lemma 3.1 shows that the class $\mathfrak{X}^{(1)}$ is a generalisation of Ayoub's class of residually commutative groups $\mathfrak{E}^{(0)}$, in the sense that when $\mathfrak{X}=\mathbb{E}$ the property residual $\mathfrak{X}$-commutativity coincides with Ayoub's property.

Lemma 3.2 (i) $\hat{\boldsymbol{P}}_{n} \mathfrak{X}(1) \leqq \mathfrak{X}^{(1)}$,

(ii) $\mathfrak{X}^{*} \leqq \mathfrak{X}^{(0)} \leqq \mathfrak{X}^{(1)}$,

(iii) $\mathfrak{X}(1)^{\dagger} \leqq \mathfrak{X}^{(1)}$,

(iv) if $\mathfrak{X}$ is $S$-closed then $\mathfrak{X}^{(1)}$ is $\langle C, S\rangle$-closed.

Proof. The proofs are routine and are omitted.

For the rest of this section we shall assume that $\mathfrak{X}=\boldsymbol{H} \mathfrak{X}=S_{n} \mathfrak{X}$.

Lemma 3.3. If $G \in \mathfrak{X}^{(1)}$ and $M$ is a minimal normal subgroup of $G$, then $M$ is $\mathfrak{X}$-abelian and $G / M \in \mathfrak{X}^{(1)}$.

Proof. (i) Suppose that $M$ is not $\mathfrak{X}$-abelian and let $x \in M-H_{1}(M: \mathfrak{X})$. Since $G \in \mathfrak{X}^{(1)}$, there exist $N \triangleleft H \triangleleft G$ such that $\mathscr{R}(x, H, N)$ and $H / N \in \mathfrak{X}$. Then $1 \neq x \in H \cap M$, so $M \leqq H$. Note that $M \cap N \in \mathscr{H}(M: \mathfrak{X})$ since

$$
M /(M \cap N) \cong M N / N \triangleleft H / N \text { and } \mathfrak{X}=S_{n} \mathfrak{X} \text {. }
$$

Hence $[x, M \cap N] \neq 1$ and there exists $b \in M \cap N$ with $[x, b] \neq 1$. By $\mathscr{R}(x, H, N)$, there exists $A \triangleleft G$ such that $\mathscr{C}(x, b, A)$. Then $1 \neq[x, b] \in M \cap A$, so that $M \leqq A$. But $M$ contains both $x$ and $b$ and thus so does $A$, contrary to $\mathscr{C}(x, b, A)$.

(ii) Suppose that $G / M \notin \mathfrak{X}^{(1)}$; then there exists $1 \neq x M \in G / M$ such that $\mathscr{R}\left(x M,{ }^{*},{ }^{*}\right)$ never holds with an $\mathfrak{X}$-factor group. Now $\mathscr{R}(x, H, N)$ for some $N \triangleleft H \triangleleft G$ and $H M / N M \in \mathfrak{X}$ by $H$-closure. Since $\mathscr{R}(x M, H M / N, N M / M)$ is false, there exists $1 \neq b M / M \in N M / M$ such that $\mathscr{C}\left(x M, b M,{ }^{*}\right)$ is always false: here we can assume $b \in N$. By $\mathscr{R}(x, H, N)$, there exists $P \triangleleft G$ such that $\mathscr{C}(x, b, P)$. Clearly $[x M, b M] \in P M / M$ so that $P M$ contains both $x$ and $b$, as $\mathscr{C}(x M, b M, P M / M)$ cannot hold. Write

$$
x=p_{1} m_{1} \quad \text { and } \quad b=p_{2} m_{2}, \quad\left(p_{i} \in P, m_{i} \in M, i=1,2\right) .
$$

Here $p_{1} \neq 1$ since $x \notin M$.

Next $\mathscr{R}\left(p_{1}, H_{2}, N_{2}\right)$ is valid for some $H_{2}$ and $N_{2}$ with $H_{2} / N_{2} \in \mathfrak{X}$. Without loss we may replace $\mathrm{H}_{2}$ by $\mathrm{H}_{2} \cap P$ and $\mathrm{N}_{2}$ by $\mathrm{N}_{2} \cap P$, using $S_{n}$-closure. Thus we can assume that $H_{2} \leqq P$ and of course $N_{2} \leqq P$. Notice that $M \cap P=1$ for otherwise $M \leqq P$ and we have $x \in P M=P, b \in P M=P$ in contradiction to $\mathscr{C}(x, b, P)$. 
$\mathscr{R}\left(x M, H_{2} M / M, N_{2} M / M\right)$ is not valid, so there exists $1 \neq d M \in N_{2} M / M$ such that $\mathscr{C}\left(x M, d M,{ }^{*}\right)$ is always false: here we can assume $d \in N_{2}$. By $\mathscr{R}\left(p_{1}, H_{2}, N_{2}\right)$ there exists $P_{2} \triangleleft G$ such that $\mathscr{C}\left(p_{1}, d, P_{2}\right)$ : here we can assume $P_{2} \leqq P$. Then of course $[x M, d M]=\left[p_{1} M, d M\right] \in P_{2} M / M$. However, as $\mathscr{C}\left(x M, d M, P_{2} M / M\right)$ cannot hold we conclude that $P_{2} M$ contains both $x$ and d. Write

$$
x=p_{3} m_{3} \text { and } d=p_{4} m_{4} \quad\left(p_{i} \in P_{2}, m_{i} \in M, i=3,4\right) .
$$

Then $p_{1} m_{1}=p_{3} m_{3}$ yields $p_{1}^{-1} p_{3}=m_{1} m_{3}^{-1} \in M \cap P=1$ so $p_{1}=p_{3} \in P_{2}$ and $p_{4}^{-1} d \in M \cap P=1$ so $d=p_{4} \in P_{2}$ : but this contradicts $\mathscr{C}\left(p_{1}, d, P_{2}\right)$ and completes the proof of the lemma.

We remark that even when $\mathfrak{X}$ is $\boldsymbol{H}$-closed, $\mathfrak{X}^{(1)}$ need not be; for by Lemma 3.2, free groups are residually $\mathfrak{X}$-commutative for any class $\mathfrak{X}$, e.g. $\mathfrak{X}=\mathfrak{F}$ but the alternating group on five letters is clearly not residually $\mathfrak{X}$-commutative.

\section{The proof of Theorem $A$}

Lemma 4.1. When $\mathfrak{X}=\boldsymbol{S}_{n} \mathfrak{X}=\boldsymbol{H} \mathfrak{X}, \mathfrak{X}^{(1)} \cap \mathfrak{M} \vee$ is $\boldsymbol{H}$-closed.

Proof. Let $M \triangleleft G \in \mathfrak{X}^{(1)}$ and suppose $G / M$ is not residually $\mathfrak{X}$-commutative. Then there exists $1 \neq x M \in G / M$ such that $\mathscr{R}\left(\bar{x},{ }^{*},{ }^{*}\right)$ is always false with an $\mathfrak{X}$-factor group; we are using ${ }^{-}$to denote images under the natural map from $G$ onto $G / M$. Since $G \in \mathfrak{X}^{(1)}$, there exist $N \triangleleft H \triangleleft G$ such that $H / N \in \mathfrak{X}$ and $\mathscr{R}(x, H, N)$. Since $\mathscr{R}(\bar{x}, \bar{H}, \bar{N})$ is false, there exists $1 \neq b \in \bar{N}$ such that $\mathscr{C}\left(\bar{x}, \bar{b},{ }^{*}\right)$ is always false and $b \in N$. But there exists $K \triangleleft G$ with $\mathscr{C}(x, b, K)$ and we may assume $K \leqq H$. Then $[x, b] \in K$ and so $[\bar{x}, \bar{b}] \in \bar{K}$. Since $\mathscr{C}(\bar{x}, \bar{K}, \bar{K})$ is false, $\bar{K}$ contains both $\bar{x}$ and $\bar{b}$, say $\bar{x}=\bar{k}$ and we may assume that $k \in K$. Now there exist $N_{1} \triangleleft P \triangleleft G$ such that $P / N_{1} \in \mathfrak{X}$ and $\mathscr{R}\left(k, P, N_{1}\right)$, and by $S_{n}$-closure we may assume $K$ contains $P$ and $N_{1}$. As $\mathscr{R}\left(\bar{x}, \bar{P}, \bar{N}_{1}\right)$ is false, there exists $1 \neq b_{1} \in N_{1}$ such that $\mathscr{C}\left(x, b_{1},{ }^{*}\right)$ is always false. Now, there exists $Q \triangleleft G$ with $\mathscr{C}\left(k, b_{1}, Q\right)$ and we may assume that $Q \leqq K$. Then $\left[\bar{x}, b_{1}\right] \in \bar{Q}$ means that $\bar{Q}$ contains $\bar{x}$ and $\bar{b}_{1}$. Put $H_{1}=P$ and $K_{1}=Q$. Then

$$
H>K \geqq H_{1}>K_{1},
$$

for $H_{1}=K_{1}$ would yield $k \in P=Q$ and $b_{1} \in N_{1} \leqq P=Q$, contrary to $\mathscr{C}\left(k, b_{1}, Q\right)$. In this way we produce an infinite descending chain

$$
H>K \geqq H_{1}>K_{1} \geqq H_{2}>K_{2} \geqq \cdots
$$

of normal subgroups of $G$, contrary to min- $n$. This completes the proof of the lemma.

Thus if $G \in \mathfrak{X}^{(1)} \cap \mathfrak{M}_{n}^{\vee}$ and $H$ is any homomorphic image of $G$ then $H \in \mathfrak{X}^{(1)} \cap \mathfrak{M}_{n}^{\vee}$ and so has minimal normal subgroup $M$, say, which is $\mathfrak{X}$-abelian by Lemma 3.3; hence $G$ is hyper- $\mathfrak{X}$-abelian. Hence (i) implies (iii) in Theorem A: that (iii) implies (ii) is obvious and (ii) implies (i) is part of Lemma 3.2. 


\section{The proof of Theorem $B$}

Throughout this section $\mathfrak{X}$ will be a variety of groups. We show that $\mathfrak{X}^{(1)}$ is a local class using a method similar to that of Theorem 10 of (6).

Let $G \in L \mathfrak{X}^{(1)}$ and pick $1 \neq a \in G$. Let $\Phi(a)$ denote the collection of all finite subsets of $G$ containing $a$ and for each $S$ in $\Phi(a)$ denote by $\Gamma(S)$ the collection of all finitely generated subgroups of $G$ containing $S$. Hence for each $H \in \Gamma(S), H \in \mathfrak{X}^{(1)}$ and so there exist $N \triangleleft K \triangleleft H$ such that $\mathscr{R}(a, K, N)$. Let $\Theta(S)$ denote the collection of all such $K$, i.e. $K \in \Theta(S)$ implies that there exists $H \in \Gamma(S)$ such that there exists $N \triangleleft K$ and $\mathscr{R}(a, K, N)$. Write

$$
\begin{aligned}
K_{a}(S) & =\cap\{K \mid K \in \Theta(S)\} \\
K_{a} & =\cup\left\{K_{a}(S) \mid S \in \Phi(a)\right\} \\
L_{a}(S) & =\cap\left\{\rho_{\nexists}^{*}(K) \mid K \in \Theta(S)\right\} \\
L_{a} & =\cup\left\{L_{a}(S) \mid S \in \Phi(a)\right\} .
\end{aligned}
$$

We now need the following two Lemmas.

Lemma 5.1. $K_{a}$ is a normal subgroup of $G$ containing $a$.

Proof. It is clear that $K_{a}$ contains $a$. Let $x$ and $y$ belong to $K_{a}$. Then we can find $S_{x}$ and $S_{y}$ in $\Phi(a)$ such that $x \in K_{a}\left(S_{x}\right)$ and $y \in K_{a}\left(S_{y}\right)$, hence $x y^{-1} \in K_{a}\left(S_{x} \cup S_{y}\right)$. Now let $g \in G$, then $x \in K_{a}\left(S_{x} \cup\{g\}\right)$ so whenever $H \in \Gamma\left(S_{x} \cup\{g\}\right), g \in H$ and as $K_{a}\left(S_{x} \cup\{g\}\right)$ is normal in $H, x^{g} \in K_{a}\left(S_{x} \cup\{g\}\right) \leqq K_{a}$, as required.

Lemma 5.2. $L_{a}$ is a normal subgroup of $K_{a}$ and $K_{a} / L_{a} \in \mathfrak{X}$.

Proof. We show first that whenever $S \in \Phi(a), L_{a}(S)$ is normal in $K_{a}(S)$. Let $m \in L_{a}(S)$ and $k \in K_{a}(S)$, then for each $K \in \Theta(S), m \in \rho_{x}^{*}(K) \square K$ and so $m^{k}$ lies in $L_{a}(S)$. Now if $x \in L_{a}$ and $y \in K_{a}$, there exist $S_{x}$ and $S_{y}$ in $\Phi(a)$ such that $x \in L_{a}\left(S_{x}\right) \leqq L_{a}\left(S_{x} \cup S_{y}\right)$ and $y \in K_{a}\left(S_{y}\right) \leqq K_{a}\left(S_{x} \cup S_{y}\right)$. Normality now follows by the opening remarks.

Now let $w\left(x_{1}, \ldots, x_{n}\right)$ be a word which is satisfied by the variety $\mathfrak{X}$ and let $k_{i} L_{a} \in K_{a} / L_{a}$ for $i=1, \ldots, n$. Then we can find $S \in \Phi(a)$ such that $k_{i} \in K$ for each $K \in \Theta(S)$. But $K / \rho_{\mathfrak{x}}^{*}(K) \in \mathfrak{X}$ and so $w\left(x_{1}, \ldots, x_{n}\right) \in \rho_{\mathfrak{x}}^{*}(K)$ for every $K$ in $\Theta(S)$ which implies that $w\left(x_{1}, \ldots, x_{n}\right) \in L_{a}(S) \leqq L_{a}$; hence $w$ is identically 1 on $K_{a} / L_{a}$ as required.

Because of Lemma 5.2 we are now able to deduce that whenever $1 \neq b \in \rho_{x}^{*}\left(K_{a}\right), b \in L_{a}$ and so for some $S \in \Phi(a), b \in \rho_{x}^{*}(K)$ for every $K \in \Theta(S)$. Let $\Phi^{\prime}(a)$ denote the collection of $S \in \Phi(a)$ such that $b \in L_{a}(S)$. Let $S \in \Phi^{\prime}(a)$; for each $K \in \Theta(S)$ we define

$$
\begin{aligned}
M_{a, b}(K) & =\cap\{M \triangleleft H \mid \mathscr{C}(a, b, M)\} \\
M_{a, b}(S) & =\cap\left\{M_{a, b}(K) \mid K \in \Theta(S)\right\} \\
M_{a, b} & =\cup\left\{M_{a, b}(S) \mid S \in \Phi^{\prime}(a)\right\} .
\end{aligned}
$$

The proof of Theorem $B$ is now completed in two Lemmas. 
Lemma 5.3. Whenever $S_{1}, S_{2} \in \Phi^{\prime}(a)$ and $S_{1}$ is contained in $S_{2}, M_{a, b}\left(S_{1}\right)$ is a subgroup of $M_{a, b}\left(S_{2}\right)$.

The proof of this lemma is obvious.

Lemma 5.4. $\mathscr{C}\left(a, b, M_{a, b}\right)$.

Proof. Clearly, in view of Lemma $5.3 M_{a, b}$ is a normal subgroup of $G$ containing $[a, b]$. Suppose both $a$ and $b$ lie in $M_{a, b}$. Then we can find $S \in \Phi^{\prime}(a)$ such that $a$ and $b$ lie in $M_{a, b}(S)$ and hence in $M_{a, b}(K)$ for every $K$ in $\Theta(S)$, which is contrary to hypothesis.

As a result of Lemma 5.4 we now see that for each $1 \neq a \in G$ there exist $L_{a} \triangleleft K_{a} \triangleleft G$ such that $\mathscr{R}\left(a, K_{a}, L_{a}\right)$ and we conclude that $G \in \mathfrak{X}^{(1)}$.

\section{Acknowledgment}

I should like to thank the referee for his useful suggestions and his improvements to some of the proofs and also the Science Research Council for their financial assistance.

\section{REFERENCES}

(1) C. Ayour, On properties possessed by solvable and nilpotent groups, $J$. Australian Math. Soc. 9 (1969), 218-227.

(2) J. R. Durbin, On normal factor coverings of groups, J. Algebra 12 (1969), 191-194.

(3) D. J. S. RobInson, Finiteness conditions for generalized soluble groups, parts 1 and 2 (Springer-Verlag, Berlin-Heidelberg-New York, 1972).

(4) O. A. Slotterbeck, Finite factor coverings of groups, J. Algebra 17 (1971), 67-73.

(5) T. E. Stanley, Generalizations of the classes of nilpotent and hypercentral groups, Math. Z. 118 (1970), 180-190.

(6) T. E. StanLey, Residual $\mathfrak{X}$-centrality in groups. Math. Z. 126 (1972), 1-5.

LeEds Polytechnic

Calverley Street

LEEDS LS1 3HE 